Investigaciones Fenomenológicas, n. 9, 2012, 269-289.

e-ISSN: $1885-1088$

\title{
INTENCIONALIDAD DE HORIZONTE Y REDUCCIÓN TRASCENDENTAL EN LA FENOMENOLOGÍA DE HUSSERL
}

\author{
Ignacio Quepons Ramírez \\ Universidad Nacional Autónoma de México/ Universität zu Köln, Alemania \\ iquepons@gmail.com
}

\begin{abstract}
Resumen: El tema principal es sugerir la continuidad entre las diferentes maneras de entender la reducción fenomenológica en la fenomenología de Husserl. A fin de presentar esta posibilidad argumento a favor de la importancia de la intencionalidad de horizonte como guía del análisis trascendental que parte de la fenomenología del mundo de la vida. Es posible entender el análisis fenomenológico como un método explicitativo que descubre paso a paso las diferentes capas de sentido, tomando como guía la descripción de la estructura del mundo como horizonte implícito de nuestras vivencias.
\end{abstract}

Palabras clave: Husserl, reducción fenomenológica, intencionalidad de horizonte, mundo de la vida.

\begin{abstract}
The main issue is to suggest the continuity between the different ways to understand the Phenomenological Reduction in Husserl's Phenomenology. In order to present for this possibility I argue for the importance of the horizon intentionality as the guide of transcendental analysis departing from the phenomenology of world of life. It is possible to understand the phenomenological analysis as an explicative method that discover step by step the different layers of sense, taking as a guide of the description the structure of the world as implicit horizon of our livedexperience.
\end{abstract}

Keywords: Husserl, phenomenological reduction, horizon-intentionality, world of life.

Como afirma Eugen Fink, en su célebre ensayo de 1933, la reducción fenomenológica es el método fundamental de la fenomenología. La forma en que se comprende y asume la reducción trascendental define la radicalidad filosófica del proyecto fenomenológico ${ }^{1}$. El tema de este trabajo es la relación entre la intencionalidad de horizonte y los diferentes accesos a la reducción trascendental; el objetivo es mostrar la coherencia entre el acceso cartesiano a la esfera trascendental y la vía del mundo de la vida, a través de la perspectiva que ofrece la interrogación regresiva de los horizontes.

1 "El método fundamental de la filosofía fenomenológica de E. Husserl es sólo y únicamente la 'reducción fenomenológica'; ella es el camino cognoscitivo del pensamiento filosófico inicial dirigido hacia el campo 'temático' de la filosofía, 'es el acceso' ('Zugang') a la subjetividad trascendental; en ella están contenidos todos los problemas de la fenomenología y los métodos especiales que están ordenados a ellos". Eugen Fink, "La fenomenología fenomenológica de Edmund Husserl ante la crítica contemporánea", Acta Fenomenológica Latinoamericana, vol. 1 (2003), p. 366. Tr. Raúl Veloso Farías 
En La Crisis de las ciencias europeas y la fenomenología trascendenal, Husserl señala que la vía "cartesiana" hacia la reducción es parcial porque lleva a la esfera trascendental como de un salto. Iso Kern, con base en esa autocrítica de Husserl, ha sugerido un cambio de rumbo en el proyecto fenomenológico que invalidaría la vía cartesiana. Frente a esta interpretación defendemos la posibilidad de entender ambos planteamientos desde una perspectiva complementaria. Para ello se destacará la importancia de la "intencionalidad de horizonte" y las implicaciones de sentido en que se constituye el mundo pre-dado de la experiencia como el hilo conductor de la coherencia entre los accesos a la reducción $^{2}$. La posibilidad que abre esta hipótesis es coherente con la idea de Eugen Fink de que la tarea de la fenomenología es la pregunta por el "origen del mun$\mathrm{do}^{\prime \prime}$.

La vía a la esfera trascendental a través del mundo de la vida, lejos de ser una renuncia al programa planteado en Ideas $I^{4}$, un programa metódico que permite acceder a la esfera trascendental por etapas de acuerdo al análisis de los horizontes implicados en el mundo pre-dado de la experiencia natural. La evidencia de la pre-dación del mundo es una evidencia implícita en toda evidencia predicativa; el carácter implícito de la mención de dicha evidencia tiene el carácter de un horizonte intencional. El análisis fenomenológico entendido como indagación del sentido implicado y sedimentado en la forma de horizontes permite alcanzar el estrato de la subjetividad trascendental como ámbito implícitamente supuesto en toda experiencia de mundo pre-dado. La subjetividad

\footnotetext{
${ }^{2}$ La importancia de la intencionalidad de horizonte en la comprensión sistemática del proyecto fenomenológico de Husserl ha sido destacada por el profesor Roberto Walton en una variedad de artículos. Mi comprensión de dicha temática en Husserl tiene una deuda grande con las investigaciones de Walton. Sólo menciono algunos trabajos que resultaron particularmente importantes en la propuesta que aquí sugerimos. Roberto Walton, Walton, Roberto, "Función y significado de la intencionalidad de horizonte", en María Luz Pintos / José Luis González López (eds.), Fenomenología y ciencias humanas, Santiago de Compostela, Servicio de Publicaciones de la Universidad de Santiago de Compostela, 1998; Roberto Walton, "Phenomenology of Horizons", en Steven Crowell / Lester Embree (eds.), The Reach of Reflection: Issues for Phenomenology's Second Century, Center for Advanced Research in Phenomenology, 2001; Roberto Walton, "Horizonticidad y juicio", Anuario Filosófico vol. XXXVII, 1 (2004). También es pertinente para el estudio de esta temática el trabajo de Sauilus Geniusas, The origins of the horizon in Husserl's Phenomenology (Doctoral Dissertation), New School for Social Research, 2008, que comprende una presentación sistemática del desarrollo de la noción de Horizonte en la obra de Husserl. Sobre el tema del horizonte y el mundo en el contexto de la fenomenología genética también es importante la última parte del estudio de Donn Welton, The Other Husserl. The Horizons of Trascendental Phenomenology, Boomington/Indanapolis, Indiana University Press, 2000, pp. 331-392.

3 "La cuestión fundamental de la fenomenología, que está a medio camino de muchos planteamientos unidos a problemas tradicionales, y en la cual se manifiesta claramente su radical oposición al criticismo, se puede formular como la pregunta por el origen del mundo". Eugen Fink, op. cit., p. 386.

${ }^{4}$ Iso Kern, "Tres caminos a la reducción fenomenológica trascendental en la fenomenología de Husserl", en Agustín Serrano de Haro (ed.), La posibilidad de la fenomenología, Madrid, Universidad Complutense de Madrid, 1997, pp. 259-294.
} 
trascendental es el horizonte "anónimo" constituyente de todo el campo de lo pre-dado, el cual, no es por principio parte del mundo constituido. Todo el mundo concreto y en general todo mundo de experiencia posible supone como horizonte de su concreción a una subjetividad que lo viva y que viva su vida en ese horizonte de mundo. La vida en su evidencia apodíctica es el horizonte coasumido en toda mención de lo que es a su vez el horizonte de todos los horizontes: el mundo mismo. Por lo tanto, la evidencia del mundo pre-dado de mi experiencia estaría fundada en la evidencia más radical: soy yo el que vivo, en mi vida, la pre-dación del mundo y dicha evidencia descubre el carácter trascendental como estrato último de la constitución del mundo. El sujeto que vive en actitud natural el mundo pre-dado de la vida es él mismo trascendental, sólo que, por así decirlo, todavía no lo sabe.

La diferencia entre este acceso a la esfera trascendental a través del mundo de la vida y la vía cartesiana es que este camino regresivo no implica la suspensión de la validez del mundo de la actitud natural "de golpe"; por el contrario, justo la propia mención de evidencia del mundo de la actitud natural la que da la pauta para inferir por implicación de horizontes el carácter necesario de una subjetividad que vive y constituye su propio mundo de sentido.

\section{LA REDUCCIÓN FENOMENOLÓGICA Y EL DESCUBRIMIENTO DEL CAMPO TRASCENDENTAL}

Según distintos estudiosos de Husserl ${ }^{5}$, existen al menos tres accesos a la reducción trascendental, 1) la vía cartesiana que practica en Ideas I y Meditaciones cartesianas, 2) la vía a través de la psicología intencional, que aparece por primera vez en las lecciones de Filosofía primera y finalmente 3) la vía a partir del análisis del mundo de la vida, que aparece en Crisis y en los parágrafos 10 a 13 de Experiencia y juicio ${ }^{6}$. Las tres vías sostienen la imposibilidad de una fundamentación radical del problema del conocimiento desde la esfera

\footnotetext{
${ }^{5}$ Cfr. Iso Kern, op. cit.; Sebastian Luft, "Husserl's Theory of the Phenomenological Reduction: Between Life-world and Cartesianism", Research in Phenomenology, vol. 34, 1 (2004) 198-234.

${ }^{6}$ Algunos han sugerido distinguir también una vía a través del "camino de las ciencias objetivas", pero hay buenas razones para afirmar que, en realidad, constituiría la misma vía que la del mundo de la vida. Las ciencias objetivas dan por sentada la validez implícita del mundo de la vida y la coherencia entre los resultados de sus investigaciones con el mundo de la vida experimentado subjetivamente. Al respecto véase Sebastian Luft, op. cit., p. 205 n.18, y Dieter Lohmar, "Trascendental Logic and Trascendental Reduction: Husserl's way into Trascendental Phenomenology through the Critic of Science", en Gary Banham (ed.), Husserl and the Logic of Experience, Palgrave, McMillan, 2005, pp. 237254.
} 
empírico-natural y abren la posibilidad de considerar a la experiencia como acceso a la esfera constituyente en una actitud distinta de la natural. ${ }^{7}$

El camino que lleva a Husserl a la dimensión trascendental es el mismo que condujo el sentido de sus Investigaciones lógicas: la búsqueda de un principio de evidencia radical como experiencia de la verdad. En cada caso, las vías de acceso a la reducción trascendental destacan el prejuicio escéptico de la idea de verdad y fundamentación que descansa en la pretensión de fundamentar el conocimiento en la posición natural-empírica. El problema de semejante empresa es doble: por un lado asume la realidad de las determinaciones del mundo externo con independencia de nuestra experiencia y por otro, cae en el círculo vicioso de suponer como condición de explicación una instancia que debería ser ella misma explicada. El primer polo del problema es un fisicalismo realista y el segundo constituye un naturalismo generalizado, que caen en el contrasentido de hacer depender la necesidad lógica de un principio neuro-fisiológico de naturaleza contingente. La primera posición asume un prejuicio dogmático y la segunda cae irremediablemente en el escepticismo. La fenomenología, a través de la reducción fenomenológica, desplaza el problema de las determinaciones intrínsecas de la realidad externa y neutraliza la dependencia de la estructura de la experiencia en su explicación natural-empírica. Esto no quiere decir que los resultados de la ciencia objetiva carezcan de valor o incluso que la interpretación natural de los procesos psíquicos no pueda ser coherente con el resultado de la investigación fenomenológica. Lo que se trata de indicar es que no es

\footnotetext{
${ }^{7}$ El fundamento y origen de la motivación filosófica a la reducción fenomenológica es el problema de la experiencia de la verdad. La verdad es el concepto fundamental de una lógica pura como teoría de la ciencia, la cual, no puede estar fundamentada en una autocomprensión que haga de ella subsidiaria de procesos psicológico-empíricos. Dicha pretensión, piensa Husserl, conduce al contrasentido de fundamentar una teoría general en una particular (la psicología) que requeriría de los supuestos que ella misma tendría que fundamentar. La fundamentación psicologista del conocimiento científico conduce para Husserl a un escepticismo. La primera salida al problema es la consideración de las vivencias desde el punto de vista de "su esencia", es decir, en abstracción ideatoria de su facticidad natural. La radicalización de esta posición constituiría una reducción fenomenológica que propone un paso anterior a la "abstracción ideatoria" consistiendo en la suspensión de la validez del mundo objetivo y la suscripción de la subjetividad a la explicación causal mundana. La primera aparición de esta teoría son las lecciones de

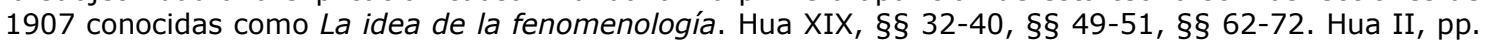
30-35 y 44-52. Una vía no implica a la otra necesariamente, aunque el sentido preciso de ambas es su complementariedad. ¿Es posible describir esencias asumiendo su validez objetiva trascendente a su formación en la subjetividad (al modo de la fenomenología realista) o bien, se puede considerar a la subjetividad trascendental fáctica sin avanzar a la consideración esencial de los sentidos descritos en las esencias? (sobre la concreción de la subjetividad trascendental: Hua I §§ 12-16 y $\S 33$ ). De manera aislada ninguno de los planteamientos es científico: el primero asume el prejuicio dogmático de la existencia de suyo de las trascendencias objetivas y el segundo no avanza a la generalización necesaria para declarar unidades universales de los conocimientos adquiridos en la descripción. No obstante, la génesis misma de las esencias remite a su constitución en la subjetividad trascendental fáctica. Con esta distinción queremos sugerir la primordialidad de la reducción trascendental como rasgo decisivo de la fenomenología frente a la "descripción de esencias de vivencias" a la manera de la fenomenología realista.
} 
ni en los presupuestos físicos ni en la explicación de la experiencia en perspectiva naturalista que se puede dar fundamento a la idea de la ciencia, que ambas explicaciones, de hecho, suponen.

La reducción trascendental es una operación de carácter metodológico, la cual está fundada en un principio de evidencia que es el hilo conductor de la forma en que se comprende el método de la reducción. Desde el punto de vista de la pura experiencia no es evidente que las determinaciones del mundo externo le sean intrínsecas (de hecho, eso no podemos saberlo) ni que exista una causalidad necesaria entre la explicación natural de nuestros procesos psíquicos y el sentido de nuestra experiencia vivida. Sin embargo, es posible identificar y universalizar estructuras necesarias de la experiencia a partir de la esfera, esa sí plena en evidencia, de la articulación de nuestras vivencias. Husserl alcanza en primera instancia el requerimiento de la reducción para acceder al campo trascendental a partir de la llamada vía cartesiana. La importancia del camino cartesiano es que destaca la apodicticidad irreductible de la vida como fundamento de toda evidencia adecuada o inadecuada. Dicha vía opera a partir de la suspensión de la validez del mundo objetivo para ganar un campo de mundo fenoménico efectivamente vivido cuya estructura esencial se trata de describir. La primera formulación del método de la "epojé" aparece en 1907 en sus lecciones conocidas como La idea de la fenomenología; la presentación más detallada del método y la determinación de la estructura del campo trascendental aparecen en 1913 en Ideas I, pero la formulación madura de la vía cartesiana se concreta en la primera de sus Meditaciones cartesianas. Las Meditaciones cartesianas tienen como antecedente público las conferencias dictadas por Husserl en París en 1929.

En la década de los años veintes Husserl exploró otras vías alternativas hacia la esfera trascendental, especialmente en sus lecciones de Filosofía primera de 1924. En estas lecciones aparece la vía conocida como vía "psicológica" a la esfera trascendental. Ahí Husserl declara que la variación de los contenidos del mundo trascendente no alteraría mis experiencias sedimentadas, mi propio pasado psíquico, en suma, mi vida. El análisis psicológico descubre un punto que es imposible explicar desde los supuestos mundanos, el cual lo lleva a considerar que la subjetividad no "forma parte del mundo". Es decir, la subjetividad no puede ser, por principio, momento no independiente del mundo, en 
la medida en que el mundo natural y sus relaciones causales son de naturaleza distinta a la de la vida de conciencia ${ }^{8}$.

Por último, tenemos el acceso a partir del descubrimiento del mundo de la vida, el cual va esclareciendo la cuestión explicando las evidencias supuestas como fondo u horizonte implícito desde la actividad judicativa de las ciencias de la naturaleza hasta el mundo vivido considerado en actitud cotidiana y precientífica ${ }^{9}$. Las ciencias, en su actividad cognoscitiva, asumen implícitamente la pre-dación del mundo de la vida como evidencia. El mundo de la vida, como mundo de la experiencia natural originaria, aparece como el fundamento olvidado de la actividad científica. La evidencia implícitamente asumida del mundo de la vida se funda su vez, de forma implícita, en la actividad constituyente de una subjetividad anónima. Somos nosotros en nuestra relación con el mundo en torno de la vida quienes constituimos implícitamente su sentido, incluso aquel en el que se mienta el mundo pre-dado de la actitud natural. Para comprender la unidad entre las vías a la esfera trascendental, detengámonos en la primera y la última.

El en caso de la vía cartesiana tenemos que el descubrimiento del carácter no-apodíctico de la evidencia del mundo $^{10}$ en la actitud natural frente a la evidencia apodíctica de la vida que vive mundo, conduce a la necesidad de suspender la validez del mundo objetivo. Esta operación metodológica consiste en la suspensión de la actitud natural que lleva a cabo los actos constituyentes de la naturaleza con sus tesis trascendentes. Es decir, se suspende la tesis de existencia en el mundo externo y la validez de la creencia en sus determinaciones como determinaciones intrínsecas, independientes de mi experiencia. Gracias a esta operación podemos dirigir la atención a nuestra propia experiencia desde una perspectiva que no asume la forma de la experiencia como subordinada al orden de la explicación causal-natural-mundana. En suma, se trata de "poner entre paréntesis" la creencia en la realidad trascendente del mundo al

\footnotetext{
${ }^{8}$ Hua VIII, pp. 126-131. Cfr. la sugerencia de Pablo Posada Varela hacia una interpretación mereológica de la reducción trascendental en "Mereología y Fantasía. El trance de manifestación de relaciones de esencia", en César Moreno Márquez / Alicia Ma de Mingo (eds.), Signo. Intencionalidad. Verdad. Estudios de Fenomenología, Sevilla, Sociedad Española de Fenomenología / Universidad de Sevilla, 2005, p. 280.

${ }^{9}$ Véase Sebastian Luft, op. cit., p. 215.

${ }^{10} \mathrm{El}$ problema del mundo y la evidencia que le corresponde es tema que requiere de cierta atención y cuidado. Aunque Husserl en repetidas ocasiones se refiere al carácter absoluto de la conciencia con independencia del mundo, esta afirmación tiene que ser matizada pues de otro modo se pierde el sentido de la correlación intencional. Sobre esta cuestión véanse Hua XXXIV, pp. 467ss y Hua XXXIX, Textos 22, 24 y 25. Véase Sebastian Luft, op. cit., pp. 203s.
} 
que están dirigidos los actos de conciencia y con ello reducir toda trascendencia a correlato de la vida de conciencia purificada. El resultado de dicha operación hace posible la apertura de la esfera de nuestra propia experiencia como acceso al ámbito constituyente de la formación de su propio sentido, y con éste, a la formación del sentido de toda la realidad asumida como puro fenómeno de sentido.

La reducción fenomenológica en la que reflexionamos sobre los propios actos de conciencia, datos de carácter absoluto e indubitable, orienta a la filosofía hacia la crítica de los intentos de fundamentarla en una ciencia natural como la psicología. Así, la investigación trascendental de la conciencia no puede ser por principio una investigación natural:

La existencia de una naturaleza no PUEDE condicionar la existencia de la conciencia, puesto que ella misma se pone de manifiesto como correlato de conciencia; la naturaleza sólo ES en cuanto se constituye en nexos regulados de conciencia $^{11}$.

La actitud fenomenológica pone de manifiesto la dependencia de toda trascendencia y todo orden de la naturaleza respecto de su correlación con la conciencia que vive sus vivencias, de lo que se sigue una primacía de la conciencia respecto del mundo ${ }^{12}$.

Todo lo trascendente, en la medida en que viene a la dación conscientemente, es objeto de investigación fenomenológica, no sólo por el lado de la CONCIENCIA DE ello, por ejemplo, de los diferentes modos de conciencia en que viene a la dación como lo mismo, sino también, aunque entretejido esencialmente con ello, como lo dado y aceptado en las daciones ${ }^{13}$.

Muchos de los análisis de la fenomenología podrían, no obstante, realizarse "sin" la reducción trascendental, pero sólo a título de análisis intenciona-

${ }^{11}$ Hua III/1, p. 109; Ideas I, p. 118. En todos los casos citamos la edición de obras completas de Husserl seguido el número de página en alemán y su correspondencia a la edición de Gaos en español. No obstante, en todos los casos en que citamos Ideas $I$ hemos utilizado la nueva versión, en proceso de publicación, del profesor Antonio Zirión a quien agradecemos el permiso para citar su traducción.

${ }^{12}$ Como hemos señalado antes, si bien esta manera de presentar la cuestión sigue especialmente la línea cartesiana de Ideas $I$, es necesario matizar dicha afirmación. Se trata de una primacía relativa pues lo que se destaca es que el acceso a la verdad del mundo es a través de la mediación necesaria de la subjetividad, entendida ella como subjetividad trascendental. Ello no quiere decir que la subjetividad sea más importante que el mundo pues lo que es la subjetividad lo es en virtud de su referencia hacia el mundo como su correlato objetivo. Subjetividad y mundo constituyen los polos del a priori de la correlación y declarar la primacía de cualquiera de los dos polos, aunque el acceso sea a través de la subjetividad, conduce a malos entendidos. Debo esta precisión a la discusión sostenida con el profesor Sebastian Luft en el verano de 2010 en Cologne-Leuven Summer School, organizado por el Archivo Husserl de la Universidad de Colonia.

${ }_{13}$ Hua III/1, pp. 159-169; Ideas I, p.170. 
les de la vida de conciencia de acuerdo a su esencia ${ }^{14}$. Sin embargo, estos análisis corresponden a una mera psicología descriptiva y no responden al problema en principio planteado por el programa de la fenomenología de realizar una crítica radical del conocimiento y sobre esta base, la construcción de la filosofía como ciencia universal fundada en evidencias absolutas. La realización efectiva de dicho proyecto es algo que todavía puede dar lugar a discusiones en un contexto más amplio, sin salirnos del marco que ofrece la propia fenomenología de Husserl, pero la radicalidad de la reducción fenomenológica es un principio irrenunciable que constituye la diferencia específica de su proyecto filosófico.

La idea de una psicología fenomenológica fue un tema que interesó a Husserl a lo largo de toda su obra filosófica; la centralidad que a veces tiene esta temática es ocasión de posibles confusiones en torno a la diferencia entre psicología y filosofía. En su artículo de la Enciclopedia Británica declara que los resultados de una psicología fenomenológica pura y una fenomenología trascendental son exactamente los mismos; incluso podría hablarse de ciencias paralelas. En los últimos parágrafos de Crisis, declara el carácter filosófico de la psicología, pero ahora poniendo en paralelo la psicología pura y lo que aquí llama "psicología trascendental" como ciencia de la subjetividad trascendental. A pesar del ocasional uso indiscriminado de la noción de psicología, sólo distinguida por el apellido (empírica, pura o trascendental), es necesario diferenciar la crítica del conocimiento que es la tarea de la fenomenología, del estudio es-

${ }^{14}$ En Ideas I por ejemplo, Husserl deja de lado la consideración trascendental para continuar la caracterización de la conciencia en la actitud natural. En §34 señala que empezamos con una serie de consideraciones en las cuales "no nos fatigaremos" con ninguna epojé. A partir de ahí hasta el $\S 48$ realiza una consideración eidética de la estructura de la conciencia en la que prescinde de la reducción fenomenológica en sentido estricto. No es hasta el inicio de $\S 48$ a $\S 49$ que se sugiere la reaparición de la cuestión, en relación al problema de la realidad del mundo. El tema de la actitud fenomenológica y la reducción fenomenológica reaparecen en $\S 50$ de manera explícita y Husserl hace el recorrido retrospectivo que recupera, ya a la luz de la reducción trascendental, lo que se obtuvo antes "sin la fatiga" de la epojé trascendental. Es verdad que los análisis llevados a cabo son psicológico-fenomenológicos porque estudian la conciencia desde su especificidad "intencional" y los contenidos que le son propios, pero ya la psicología descriptiva de Investigaciones Lógicas realizaba estos análisis sin la reducción trascendental. Esto no quiere decir que los resultados de dichos análisis difieran de la consideración en la actitud trascendental $I_{-}$; de hecho, la perspectiva trascendental mantiene los resultados pero mediante un cambio de perspectiva reinterpreta los contenidos asumidos por la posición de la psicología intencional en términos de la constitución trascendental. Esta posibilidad funda el camino de la reducción psicológica o a través de la psicología que explora en Filosofía primera, el artículo de la Enciclopedia Británica, y que expone con detalle al final de Crisis (véanse $\S \S 56 s s$ ). Esta "posibilidad" de realizar los análisis de la estructura intencional, por así decir, en "off" sin la ejecución de la reducción trascendental y describir los correlatos sin la suspensión de la validez del mundo va a permitir que el mundo de la vida, como fundamento de las ontologías formal y regionales, sea el hilo conductor legítimo de los análisis constitutivos y resuelve el problema del aparente "salto" a la subjetividad trascendental que es el problema que Husserl le encuentra a su "camino cartesiano" en la reducción trascendental, según su propia declaración en Crisis § 43. Véase también nuestra nota 3 , in supra. 
pecífico de las formas de la subjetividad mundana concreta, asunto de la psicología.

En el fondo se trata de una diferencia de actitud y proyecto; la fenomenología trascendental en cuanto depuración y crítica de la experiencia puede echar mano de los resultados de una psicología pura por cuanto la tarea de la crítica de la experiencia descubre estructuras universales que son estructuras de la vida psíquica de los sujetos concretos. Si tomamos la vida individual del sujeto en su especificidad psíquica, dichas estructuras no requieren ser explicadas por la causalidad natural y por tanto pueden ser utilizadas como orientación de análisis fenomenológicos. No obstante, en modo alguno debe confundirse a la fenomenología con una caracterización psicológica de los procesos subjetivos pues con ello se pierde la perspectiva de su talante filosófico y no se atiende a su pretensión de fundamentar una teoría de la constitución universal de todo sentido trascendente en la inmanencia de la vida ${ }^{15}$.

Aunado a esta cuestión está el problema al que Husserl se confrontó al final de su vida en torno a la relación entre los resultados o efectos "fácticos" de la reducción y sus correlatos histórico-temporales sobre el mundo concreto una vez efectuada. Es decir, ¿qué ocurre con un ser humano fáctico que se ha dado cuenta de que la actitud trascendental es ella misma una de las potencialidades antes implícitas de su propia actitud natural? Para Husserl, la reducción trascendental y su "tener lugar" en la historia es ocasión de una reconfiguración "efectiva", "fáctica" en el curso histórico de la humanidad ${ }^{16}$. Dicho problema nos coloca en la aparente paradoja de una subjetividad que en el camino a su autorealización se separa del mundo de la actitud natural; pero al final no "vivimos" todo el tiempo en actitud trascendental sino que es el mundo natural de

\footnotetext{
${ }^{15}$ Dicho talante en principio no es más que una pretensión que requiere ser evaluada con detalle. En cualquier caso el asunto fundamental es entender que la crítica de este proyecto requiere ser realizada en la arena de la filosofía y no en el de la consideración psicológica.

${ }^{16}$ Al final del $\S 35$ de Crisis Husserl señala la modificación radical en la esfera "personal" del que ejecuta la reducción fenomenológica, la cual compara con una conversión religiosa. "la actitud total fenomenológica y la epojé pertinente está esencialmente llamada a obrar, en primer lugar, una transformación personal que tendría que ser comparada con una conversión religiosa, la que más allá de eso entraña en sí el significado de la más grande transformación existencial que se ha propuesto al ser humano como ser humano". Hua VI, p. 140 Crisis; op. cit., p. 179. Es así que la reducción fenomenológica tiene efectos en la "esfera de la actitud natural" y no sólo pone de manifiesto el carácter constituyente de la subjetividad concreta, que es ella misma, la del sujeto-humano, trascendental, sino que, al hacerlo, transforma la comprensión "existencial" del sujeto humano respecto de sí mismo y su mundo entorno. Viendo "las mismas cosas", después de la reducción, por así decir, ya no se ven las cosas de la misma manera. Husserl se ocupó largamente de esta cuestión en sus manuscritos tardíos. El texto 22 de Hua XXXIV del 28 de Noviembre y principios de Diciembre de 1931 retoma el tema de la relación entre la reducción fenomenológica y su acontecimiento histórico-humano. Véase el planteamiento de la cuestión en Hua XXXIV, p. 312.
} 
la vida del que nos ocupamos y sobre el cual, paradójicamente, tiene lugar la propia ejecución de la reducción trascendental ${ }^{17}$.

\section{LA VÍA CARTESIANA Y LA VÍA A TRAVÉS DEL MUNDO DE LA VIDA VISTAS A PARTIR DEL ANÁLISIS DE HORIZONTES}

Para comprender el sentido de la autocrítica que realizó Husserl de su propio proyecto, es necesario esclarecer en qué medida la parcialidad de la vía cartesiana se complementa con la vía a través del mundo de la vida. En realidad la diferencia entre la vía cartesiana y la vía a través del mundo de la vida responde a dos momentos del proyecto fenomenológico. El primer momento está dirigido a destacar la vida concreta como evidencia apodíctica y el segundo realiza la restitución del mundo efectivamente vivido en sus relaciones cotidianas como el auténtico correlato de nuestras vivencias. Es así que la reducción fenomenológica puede ser entendida como una crítica de los contenidos de la experiencia en la que se trata de reclamar el principio de evidencia fundamental que ofrece la confirmación de la verdad en su sentido más propio. Tanto en la vía cartesiana como en la vía a través del mundo de la vida es la evidencia el hilo conductor del desarrollo metódico de la operación fenomenológica fundamental. El problema es que la fenomenología no se contenta con declarar la apodicticidad del ego-cogito-cogitatum sino que aspira a la explicitación del sentido objetivo como un proceso de constitución en diferentes estratos y niveles de la experiencia. La explicitación de las estructuras universales de la vida de conciencia sólo puede llevarse acabo como la explicitación de la forma en que la conciencia se refiere al mundo al que está dirigida y por el cual adquiere ella misma consistencia. Entonces la pregunta es, ¿cuál es el mundo al que está dirigida nuestra actividad intencional? Si toda actividad judicativa supone implícitamente un mundo de objetos al cual están referidos sus juicios tenemos que nuestra actividad judicativa asume siempre la evidencia de un mundo dado previamente a nuestros juicios. La vía del mundo de la vida explora la estructura intencional en que se constituye el mundo pre-dado.

\footnotetext{
17 Sobre este tema véase Sebastian Luft, Subjectivity and Lifeworld in Trascendental Phenomenology, Evanston IL, Nortwestern University Press, 2011, pp. 103-125.
} 
El problema de esta vía "cartesiana", como Husserl señala en Crisis, es que nos lleva a la subjetividad trascendental como de un salto, y en cierto modo, nos presenta la subjetividad trascendental misma como un polo vacío y abstracto.

Al pasar observo aquel camino, demasiado corto, recorrido hacia la epojé trascendental en mis Ideas para una fenomenología pura y la filosofía fenomenológica, camino que yo, denomino "cartesiano" [...] que tiene la gran desventaja de conducir al ego trascendental como de un salto, éste empero, ya que debe carecer de toda explicación previa, pone a la vista un vacío de contenido aparente frente al que uno se halla perplejo en cuanto qué se debe ganar con eso y completamente acerca de cómo, a partir de allí se deba alcanzar una nueva ciencia de los fundamentos de tipo totalmente nuevo, decisiva para una filosofía. ${ }^{18}$

No obstante, la potencia de dicha vía está en que descubre una evidencia apodíctica e irrenunciable que es la comparecencia de nuestra propia vida, ella misma vivida sin posibilidad de duda como evidente. En Meditaciones cartesianas Husserl vuelve sobre dicha vía para presentar el proyecto fenomenológico; a diferencia de Ideas $I$, en esta obra aparece una reconsideración de la evidencia cartesiana con un modelo de subjetividad que ya no es el polo vacío sino una mónada concreta cuya vida se abre en el modo de un horizonte ${ }^{19}$. Esto queda claro desde los primeros parágrafos de la obra, donde distingue la evidencia apodíctica del yo en cada momento de su vida del carácter inadecuado

${ }^{18}$ Husserl, La crisis de las ciencias europeas y la fenomenología trascendental, Buenos Aires, Prometeo Libros, 2008, p. 196. Trad. Julia Iribarne. [Hua VI, p. 158].

${ }^{19}$ Esta modificación se debe, según Iso Kern, a la insuficiencia de la vía cartesiana para acceder a la subjetividad concreta que es siempre con su propia historia. La inclusión de esta nueva forma de entender la subjetividad en su concreción histórica, con su vida abierta en horizontes predelineados y sus sedimentos de habitualidad en Meditaciones cartesianas es la prueba de la vigencia de la vía cartesiana, con las modificaciones que Husserl integró de sus reflexiones sobre la subjetividad trascendental de sus lecciones de los años veintes sobre Filosofía primera. Husserl mismo parece dar la pauta de la reintegración y continuidad del residuo de la reducción cartesiana con la subjetividad como mónada concreta. Resulta interesante que Kern no repara en este problema y sí da excesivas e injustificadas concesiones a la crítica de la teoría de la intersubjetividad de la Quinta Meditación, cuando afirma que "Por el camino cartesiano se alcanzaría la subjetividad ajena solamente como 'mero fenómeno'. Con razón se reprochó a Husserl en ciertas críticas que su filosofía fenomenológica no era capaz de tener en cuenta el ser propio de la subjetividad ajena. Pero esta crítica -así debe decirse anticipadamente otra vez- sólo tiene razón desde el punto de vista del camino cartesiano, que no alcanza el sentido propio de la reducción fenomenológica trascendental de Husserl" (Iso Kern, op. cit., p.267). Las precisiones a la teoría de la intersubjetividad a partir del horizonte del mundo de la vida no invalidan el carácter apresentativo en el encuentro con el otro, el otro siempre se manifiesta en su dimensión como alter ego en la forma de una apresentación; no podría ser de otra manera por razones esenciales. Kern confunde, en su concesión a los críticos de Husserl, entre el modo de aparición del otro y la evidencia de la subjetividad del otro gracias a la remisión del horizonte al mundo de la vida. Pero, en cualquier caso, el problema nunca fue demostrar si el otro "existe" o "no", sino describir los modos en que la subjetividad se refiere intencionalmente al otro. Lo que Husserl confirma con la vía cartesiana que resulta en la apresentación del otro, es el carácter intransferible de mis vivencias a él y mi imposibilidad de vivir "sus vivencias" como mías. Merleau-Ponty, que se cuenta entre los críticos de la teoría de la intersubjetividad de Husserl reconoce que hay un "solipsismo vivido que no es superable" (Maurice Merleau-Ponty, La phenomenologie de la perception, Paris, Gallimard, 1945, p. 411). La "superación" de este solipsismo vivido es la aniquilación del sentido del otro como "otro yo". 
de dicha evidencia respecto de la totalidad de la vida de la subjetividad que se extiende en un horizonte abierto.

La adecuación y la apodicticidad de una evidencia no tienen por fuerza que ir mano a mano. Quizás se hizo esta observación justamente para el caso de la experiencia trascendental del yo. En esta experiencia es el ego originariamente accesible a sí mismo. Pero esta experiencia sólo ofrece, en todo caso, un núcleo de realidad experimentada de un modo "propiamente adecuado". A saber: la actualidad viva del yo, que expresa el sentido gramatical de la proposición ego cogito, mientras que más allá de esta actualidad sólo se extiende un indefinido horizonte universal y presuntivo, un horizonte de realidad propiamente no experimentada, pero necesariamente coasumida [Mitgemeintem]. A este horizonte pertenece el pasado del yo, las más de las veces completamente oscuro, pero también la facultad trascendental propia del yo, y las cualidades habituales en cada $\operatorname{caso}^{20}$.

En estricta analogía con la percepción exterior, la cual ciertamente no es apodíctica, pero que entrega la cosa misma con certeza, la evidencia de la experiencia trascendental del yo existo trascendental, abarca

la indefinida universalidad de un horizonte abierto inherente a él. La realidad [Wirklichsein] de la base primera en sí del conocimiento es según esto absolutamente firme; pero no así sin más aquello que define de una manera más precisa la realidad de esta base, ni aquello que todavía no está presente ello mismo, sino que sólo está presumido, en el curso de la evidencia viva del "yo soy" [Ich bin.$^{21}$

Es así que la vida permanece abierta en cuanto a los contenidos no presentes de su pasado y su porvenir en la forma de un horizonte que, de acuerdo a su propio sentido, predefine la manera en cómo se realiza en su curso la vida de la subjetividad. Se trata en este caso de una evidencia inadecuada de la subjetividad como proceso abierto de experiencias en cuanto a su totalidad, pero respecto a cada momento efectivamente vivido hablamos de apodicticidad a cabalidad. Las Meditaciones cartesianas corrigen al menos en este punto la parcialidad o insuficiencia de la reducción fenomenológica como fue presentada en Ideas $I$ pues nos entrega no un polo yo vacío como residuo de la operación reductiva, sino que entrega la concreción de una vida fluyente y concreta que a

\footnotetext{
${ }^{20}$ Hua I, p. 62; Meditaciones cartesianas, México, Fondo de Cultura Económica, 1985. p.64 ss. Tr. José Gaos / Miguel García Baró.

${ }^{21}$ Hua I, p. 62; Meditaciones cartesianas, p. 65.
} 
su vez cumple el principio de apodicticidad que exige su principio radical de evidencia de la vía cartesiana ${ }^{22}$. Aquí hay que observar además que la idea de la vida como "horizonte abierto" de evidencia inadecuada, no obstante, apodíctica encuentra su estricto paralelismo con el "horizonte de horizontes" que es el mundo. La diferencia entre ambos, en esta perspectiva, es que la vida en su vivir es aunque evidentemente inadecuada siempre apodíctica, y el mundo es un horizonte inadecuado y no apodíctico en su evidencia ${ }^{23}$.

La exploración de la subjetividad trascendental es estudiada en Meditaciones cartesianas a la luz del problema de la constitución trascendental. En esta obra, una vez efectuada la reducción fenomenológica trascendental, sigue la explicitación del proceso constitutivo de la realidad a partir de las estructuras universales de la subjetividad, las cuales, se descubren en el mismo proceso explicitativo de las unidades que constituye. Aquí Husserl indica que el hilo conductor del análisis trascendental constitutivo es la "cosa", es decir, el objeto en cualquiera de sus determinaciones. Esta afirmación es decisiva para entender el sentido de la propia reducción trascendental; son la objetividad mentada en cuanto tal y el cómo de su aparición los que van a dictar la pauta de cuáles actos u operaciones intencionales supone su aparición.

Desde el punto de vista de la constitución la pregunta es nuevamente, ¿qué cosas, qué objetividades son las que serán el hilo conductor del análisis trascendental? En Meditaciones cartesianas Husserl echa mano de su ontología formal, y sus ontologías regionales como los hilos conductores de los sistemas constitutivos universales. No obstante, una de las posibles dificultades que enfrenta esta consideración, es que aunque se trate de ontologías "puras", es decir, ciencias de esencias, no son por ello en principio menos dogmáticas respecto de su fundamentación trascendental ${ }^{24}$. En otras palabras, el problema es qué evidencia y qué sentido de evidencia tenemos de ellas, pero sobre todo, en qué medida esas evidencias de la esfera universal de esencias no están ellas mismas fundadas en la esfera relativa, concreta del mundo circundante pre-

22 El tema de la "epojé" cartesiana y sus consecuencias tiene muchas aristas y una consideración más amplia del problema debería tomar en cuenta otras críticas de Husserl a su planteamiento cartesiano. Sobre este punto véase por ejemplo el texto 28 de Hua XXXIV "Crítica de la epojé cartesiana", del 10 de Febrero de 1933. La vía que ofrece aquí precisamente enfatiza la importancia del análisis de horizontes como acceso a la reducción.

${ }^{23}$ Cfr. infra n.11.

${ }^{24}$ Hua I, §§ 28-29, pp. 96-99. 
científico del mundo de la vida. Ésta es la apuesta de Crisis, tomar el mundo de la vida como él mismo, hilo conductor del análisis trascendental.

El retorno al mundo de la vida se realiza explicitando el mundo pre-dado de la experiencia como evidencia asumida implícitamente por la idealización científica. Esta explicitación no se detiene en un señalamiento de la pre-dación, sino que descubre el mundo de la vida como mundo de experiencia. En este punto, Husserl detaca la importancia de la determinación de las estructuras del mundo de la vida como hilo conductor del análisis trascendental. La vía de exploración fenomenológica de dichas estructuras es su exploración en términos del análisis de los horizontes implicados en toda vivencia concreta, así como la implicación de todo contenido presente al nexo que lo une a toda la historia de la subjetividad. La explicitación de esta génesis es la explicitación de la historia implícita de la subjetividad que funda los modos en que el mundo es pre-dado. Estas formas son operaciones y rendimiento, efectuaciones sedimentadas en la experiencia que se explicitan en el análisis de lo que está asumido implícitamente. Pero no se trata aquí de cualquier asunción sino de la co-asunción de una evidencia no tematizada ${ }^{25}$.

La evidencia no tematizada, dada por supuesta en su obviedad, es la evidencia de la pre-dación del mundo como un mundo pre-dado para mí. Yo, el yo que vive este mundo asumido en la actividad judicativa como previamente dado a mis juicios, asumo a su vez esa pre-dación como pre-dada para mí de modo implícito, es decir, no reflexivo ni patente. Dicho de otra forma: en el momento de su ejecución no tematizo mi actividad constituyente de mundo. La reflexión sobre mi actividad intencional y el descubrimiento de que aquello que soy es un campo unificado y coherente de vida que instituye sentido, puede ser alcanzada en una reflexión psicológica, es decir, en una reflexión que asume la creencia en que yo, en tanto sujeto reflexionante, soy también un sujeto en un mundo terminado $y$, en ese sentido, un sujeto ya constituido.

La reflexión psicológica, llevada a sus últimas consecuencias, no puede suponer la validez del mundo ni remitir su vida a las explicaciones mundanas puesto que mi vida, con todos sus contenidos, se mantiene exactamente igual

${ }^{25}$ Ésta es la vía que sugiere el $\S 11$ de Experiencia y juicio. Edmund Husserl Erfahrung und Urteil, Hamburg, Classic Verlag, 1969, pp. 47-49. El análisis de las estructuras del mundo pre-dado fue objeto de estudio en los manuscritos de investigación del legado póstumo de Husserl. Cfr. el texto n.4 de Hua XXXIX, pp. 26-40. 
en la suspensión del mundo. La reflexión psicológica ofrece a la subjetividad una evidencia que no puede explicar desde los supuestos explicativos mundanos. Antes bien son dichas explicaciones mundanas las que requieren fundamentación, y esto quiere decir que es necesario explicitar los contenidos asumidos implícitamente por las ciencias objetivas como parte de un mundo vivido en referencia a la experiencia de un "yo" concreto. Pero esta explicitación no puede suponer esta vida yoica como un yo psicológico y por tanto mundano, por lo cual debe afirmarse que la subjetividad no es una parte no-independiente del mundo. La subjetividad que la reflexión psicológica progresiva lleva hasta sus últimas consecuencias excede sus propios supuestos mundanos y mienta implícitamente la esfera trascendental.

La confirmación de esta evidencia permite "suspender" la validez de la tesis de realidad. En el marco de dicha epojé se abre el campo total de la experiencia constitutiva. La exploración propuesta aquí a modo de hipótesis sugiere una posibilidad de pensar la coherencia entre la vía cartesiana de la reducción trascendental y la vía a partir del mundo de la vida.

La clave que da sentido al acceso a través del mundo de la vida a la esfera trascendental es el análisis de horizontes. La intencionalidad de horizonte no es una mera cualidad de conciencia entre otras sino que implica un método nuevo para el análisis intencional ${ }^{26}$. La explicitación de los objetos intencionales se realiza a partir del análisis de lo que está mentado implícitamente en su dación, dicha mención implícita es un horizonte de remisión hacia los momentos que están supuestos como capas necesarias en su formación. Husserl le llama a este procedimiento "genealogía" y consiste en una pregunta que interroga por aquello que está supuesto o implicado en la mención actual ${ }^{27}$.

Así como la lógica formal supone la dación de su objeto sin tematizarlo, como dice André de Muralt "supone la evidencia donadora de su objeto" y esto significa que "implica, ejerce de manera anónima la evidencia donadora de su objeto propio sin reflexionar explícitamente sobre ella. Presupone una temática trascendental. Admite los resultados de la evidencia originaria y donadora como

${ }^{26}$ Hua I, p. 19

27 Ejemplos de este procedimiento es la introducción de Experiencia y juicio donde interroga la genealogía de la lógica, o bien, en los parágrafos 4 y 5 de la Primera de las Meditaciones cartesianas donde descubre la implicación teleológica de todo juicio actual, como remisión hacia la verdad en su posibilidad, lo que llama aquí, el ideal de la ciencia como fenómeno noemático. En ambos casos Husserl se pregunta sobre aquello que está asumido de manera implícita en la actividad de juzgar. 
si estuvieran dados en sí, absolutamente, mientras que ellos remiten como index a los a priori subjetivos y constitutivos correspondientes" ${ }^{28}$, así debemos decir que la reflexión psicológica explora las formas de referencia al mundo predado de la vida. No obstante, en el análisis de sus propias implicaciones descubre sus límites, pues el análisis revela que esos modos de referencia intencional no son y no pueden ser "meramente psicológicos" en sentido mundano sino que son los modos en que la subjetividad trascendental constituye el mundo de la vida ${ }^{29}$.

En general esta idea de subjetividad trascendental es una suerte de autoconciencia en la que la subjetividad reconoce, en una "superación" de la perspectiva naturalista, su verdadera esencia trascendental y constituyente ${ }^{30}$.

El acceso a la esfera trascendental desde el mundo de la vida puede ser explicado a partir de la siguiente hipótesis de investigación: la estructura del mundo de la vida implica, como horizonte implícito (potencial), la subjetividad trascendental constituyente tomada como una evidencia asumida de manera implícita al punto de la imposibilidad de revelarse a la reflexión natural. La comparecencia efectiva de la subjetividad trascendental al investigador es sólo posible por la reducción trascendental, pero la esfera trascendental ya está tácitamente asumida como una forma última de evidencia manifiesta en la obviedad de que la vida es "mía" y es efectivamente vivida por mí. Cualquiera que viva en la actitud natural vive en esta evidencia sin llevarla necesariamente a sus últimas consecuencias. Sólo la reducción trascendental destaca esta evidencia en su justa dimensión y abre el marco depurado para las investigaciones fenomenológico-constitutivas.

Lo que la genealogía de la lógica pone de manifiesto no es sino el razonamiento de quien se conduce ateniéndose a la evidencia, interrogando su pro-

${ }^{28}$ André De Muralt, La idea de la fenomenología: el ejemplarismo husserliano, México, U.N.A.M., 1963, p. 222. Tr. Ricardo Guerra.

${ }_{29}$ En realidad siempre y en cada caso se trata de una y la misma subjetividad concreta de la experiencia, pero en una actitud que asume sus operaciones de manera diferente. Esto es lo que le permite a Husserl afirmar el paralelismo entre la psicología fenomenológica y la fenomenología trascendental. Se trata en efecto de un paralelismo en cuanto al descubrimiento de las formas de referencia intencional, pero difieren en los alcances y pretensiones de una y otra empresa científica. El análisis psicológico, en el mejor de los casos, es una propedéutica o permite reconocer ciertas pautas de orientación de un análisis fenomenológico estricto.

${ }^{30}$ Es necesario repensar la relación entre Hegel y Husserl. Más allá de los propios prejuicios de Husserl hacia la filosofía de Hegel, al final de su vida se acerca mucho a la consideración histórica de la fenomenología como superación histórica de sus antecedentes en la filosofía moderna. Cierta lectura "fenomenológica" de la Fenomenología del Espíritu es un buen hilo conductor para pensar la relación entre la subjetividad natural y la subjetividad trascendental así como el sentido de su historicidad. 
pia experiencia por los sedimentos implicados en su actividad judicativa y prejudicativa. Aquel que sea consecuente con la pauta del análisis de las evidencias implícitas en la experiencia, tendría que reconocer por necesidad la evidencia final que sólo muestra la reducción: la vida concreta como instancia constituyente universal de todo sentido ${ }^{31}$.

La ventaja de esta vía frente a la vía cartesiana, es de orden metodológico. No suspende de un solo golpe "el mundo" como mera trascendencia objetiva, sino que, gracias a la explicitación de horizontes implícitos, permite comprender la idea de mundo en un sentido diferente: como horizonte de sedimentación de operaciones implícitamente asumidas en la tesis natural del mundo, que en última instancia son las operaciones de la subjetividad trascendental cuya evidencia es una potencialidad de la propia vida en la actitud natural ${ }^{32}$.

El mundo, con todos sus contenidos tal y cómo se ofrecía ya dado de antemano a la experiencia, se mantiene exactamente igual una vez realizada la reducción fenomenológica. En realidad la actitud natural constituye la evidencia presupuesta y obvio punto de partida de cualquier empresa científica; en esa actitud el mundo se nos ofrece originariamente. La subjetividad a partir de su intrincada red de operaciones intencionales ofrece al mundo y su sistema de referencias significativas como dado de antemano, de manera anónima. El sujeto de la actitud natural es él mismo trascendental, sólo que en la actitud natural lo ignora. De esta manera, el resultado del análisis constitutivo no es la "generación" o "reinvención del mundo" como si el sujeto reducido comenzara "desde ceros" a producirlo, sino que el mundo ya estaba dado y fue dispuesto de tal y cual manera que la reducción no hace más que explicitar el sentido de su configuración actual en relación a la actividad que pre-reflexivamente ya había realizado la subjetividad trascendental ${ }^{33}$.

El programa de Crisis en realidad se sostiene en dos premisas que ya habían sido enunciadas, o que al menos podrían rastrearse hasta Investigaciones lógicas e Ideas $I$ : la primera es que toda intuición categorial, en este caso, las correspondientes a la evidencia de las ontologías o sistemas de esencias, está fundada en una intuición "sensible". La evidencia del mundo sensible pre-

\footnotetext{
${ }^{31}$ Cfr. Hua XVII, § 12 y $\S \S 86-86$, y Dieter Lohmar, op. cit., p. 244.

32 Véase Sebastian Luft, "Husserl's Theory of the Phenomenological Reduction: Between Life-world and Cartesianism", p. 218.

${ }^{33}$ Cfr. Crisis, § 29 y § 30.
} 
dado y asumido como evidente por las ciencias y sus idealizaciones está ya anunciada en ese carácter fundado de toda intuición categorial en intuiciones sensibles. Todo el programa de la fenomenología genética queda anunciado en la sexta de las Investigaciones lógicas con esa declaración ${ }^{34}$. La segunda premisa es la importancia que va a tener ya en Ideas I la noción de "actitud natural", especialmente cuando Husserl declara que la reducción sólo suspende la tesis de realidad y validez de los hechos de la actitud natural pero no su contenido fenoménico. El mundo de la actitud natural es exactamente el mismo que el mundo de la actitud trascendental, y la subjetividad concreta, la mónada, era ella misma ya trascendental sólo que antes de la ejecución de la reducción, por así decirlo, no lo sabía. Sus operaciones constitutivas ya estaban operando y ofreciéndole el mundo y su sentido como entorno pre-dado antes de que reflexionara fenomenológicamente sobre la naturaleza de dichas estructuras ${ }^{35}$.

La reducción trascendental es la declaración filosófica de alcances ontológicos que destaca que la estructura de sentido, en la cual se configura nuestra aprehensión de toda trascendencia, depende de una instancia que no puede ser explicada de acuerdo a la causalidad intra-mundana y en ese sentido está "afuera del mundo". No obstante, y aquí es donde está la cuestión interesante, no puede descubrir ninguna de sus estructuras constituyentes sin el "mundo", comprendido en sentido amplio, en el cual tienen lugar sus operaciones como horizonte universal.

El idealismo trascendental propuesto por Husserl implica un énfasis en la subjetividad, mas no un desapego de la realidad concreta y a todo aquello a lo que habitualmente llamamos mundo pues es en este mismo mundo de la vida cotidiana, donde la subjetividad trascendental descubre sus propias estructuras constituyentes, incluso en un sentido más concreto que el de las ciencias disciplinares empíricas. El resultado es una teoría universal de las estructuras de la conciencia y su correlato objetivo es una ontología trascendental universal ${ }^{36}$. El vínculo e hilo conductor de todo el análisis es esa peculiaridad de la vida de conciencia que es la "intencionalidad", el a priori de la correlación universal y

${ }^{34}$ Hua XIX/2, §§ 46-48.

${ }^{35}$ Como menciona Sebastian Luft en "Husserl's Theory of the Phenomenological Reduction: Between Life-world and Cartesianism" (p. 204, n.4), "El correlato del mundo de la vida es el modo de vivir en el cual el mundo de la vida es el horizonte para cualquier acción: la actitud natural". El correlato noético cuyo rendimiento es el mundo de la vida es justamente la actitud natural. Es necesario distinguir esta noción de "actitud natural" de la "actitud naturalista" que aparece por ejemplo en Ideas II.

${ }^{36}$ Hua I, p. 181. 
no nos desapegamos del sentido de Husserl si lo llamamos también: el logos concreto de la experiencia viva.

La investigación radical acerca de los primeros principios de la constitución universal no es sino la pregunta por el origen mismo de la intencionalidad, la cual, en lo que refiere a su correlato objetivo es el "origen del mundo" mismo, al que se refería Eugen Fink. Sin la reducción trascendental todo el trabajo descriptivo de la fenomenología se queda en una mera psicología o "análisis reflexivo" de escaso interés para un proyecto que aspira a aclarar el sentido de una filosofía con aspiración a Filosofía primera ${ }^{37}$.

Si el resultado de la fundamentación trascendental de la lógica formal es una lógica trascendental, y el correlato objetivo de la lógica formal es una ontología formal (es decir, una teoría universal del "algo en general" y sus formaciones), el correlato objetivo de la lógica trascendental sería una ontología trascendental. Ésta, junto con la fundamentación trascendental, que no es sino la aclaración del sentido de la experiencia implicada en la mención de las objetividades de las ontologías regionales en actitud trascendental, da como resultado una ontología trascendental universal. Tal ontología se expresaría desde el punto de vista estático como el a priori universal de la correlación y desde la perspectiva de la génesis, como la interrogación radical por la formación originaria de las objetividades, que en lo radical, coincide con la interrogación por el origen de la intencionalidad, el origen del logos universal, en nuestra experiencia concreta.

\footnotetext{
${ }^{37}$ Eso no quiere decir que no carezcan de valor científico las investigaciones dirigidas a trazar las vías de relación entre la tradición fenomenológica y la práctica fenomenológica comprendida como análisis reflexivo; esta ha sido la propuesta, por ejemplo, de Lester Embree como vía de acercamiento a otras disciplinas científicas. Pero, cómo él mismo ha destacado, estas tareas son llevar a la fenomenología "más allá de la filosofía", lo cual dista de ser el proyecto del propio Husserl. A mi juicio, tales tareas son en todo caso análisis preparatorios para la aclaración fenomenológica de los conceptos fundamentales de las ciencias empíricas, lo cual es todavía una empresa por realizar. Los análisis reflexivos y las consecuentes descripciones "fenomenológicas" de aspectos de la vida concreta son propedéuticos en el estudio fenomenológico, pero sólo tienen auténtica radicalidad filosófica cuando se descubre a través de ellos el sentido de los problemas fundamentales. Una prueba de este procedimiento es el ensayo de Agustín Serrano de Haro, La precisión del cuerpo, análisis filosófico de la puntería, el cual, lejos de ser una mera descripción del fenómeno del "apuntar", avanza desde ahí a un posicionamiento frente a Heidegger y las implicaciones del problema de la percepción y la trascendencia del mundo. Sin la referencia a los problemas fundamentales corremos el riesgo de hacer de la fenomenología una mera descripción de curiosidades subjetivas, una filosofía de estampitas que se pierde en la dispersión. La insuficiencia de la pura psicología descriptiva para los fines del proyecto fenomenológico, incluso cuando es paralela en cuanto descripción rigurosa de la vida de conciencia, es declarada por el propio Husserl en el $\S 14$ de Meditaciones cartesianas, donde llama a la distinción entre psicología descriptiva y fenomenología trascendental pues la confusión entre ambos enfoques corre el riesgo del "psicologismo trascendental" que hace imposible una auténtica filosofía.
} 


\section{CONCLUSIONES: REDUCCIÓN TRASCENDENTAL Y EL A PRIORI DE LA CORRELACIÓN}

En este ensayo estudiamos la posibilidad de una coherencia entre el acceso cartesiano y el acceso a través del mundo de la vida al campo trascendental. De igual modo planteamos la cuestión del problema de la constitución trascendental a la luz del hilo conductor de las ontologías. Sugerimos la posibilidad de entender la explicitación del sentido de las ontologías, como índices de sistemas de constitución, en la explicitación de sus resultados en la génesis trascendental que comienza con la remisión de todo sistema categorial de objetos al mundo pre-dado de la vida. Este mundo asumido de manera implícita por las ciencias objetivas y por la ontología formal misma, implica a su vez la pre-asunción de la subjetividad trascendental constituyente de sentido. El mundo de la vida aparece al final como el rendimiento de las operaciones de una subjetividad anónima que ya operaba implícitamente en la pre-dación del mundo.

En este ensayo sólo alcanzamos a explorar de forma preliminar la coherencia entre los accesos a la esfera trascendental, destacando la importancia de la intencionalidad de horizonte como procedimiento metódico de exploración de la experiencia que permite referirse a la esfera trascendental como un horizonte implícito de operaciones subjetivas cuyos efectos se viven en la actitud natural de forma implícita. La diferencia entre la actitud trascendental y la actitud natural es justo una diferencia de actitud, de asunción de la tesis de existencia del mundo que tiene, en tanto actitud, una disposición a la acción en consecuencia (de ahí que Husserl considerara la reducción con alcances existenciales). Pero el mundo en cuanto a sus contenidos fenoménicos, es siempre en un caso y en otro el mismo.

La reducción trascendental es un acceso al mundo concreto de la experiencia; éste mismo mundo que vivimos todos los días y que está supuesto como referente implícito de todas las explicaciones científicas, pero visto, gracias a la reducción, en el mayor grado de concreción experiencial posible ${ }^{38}$.

\footnotetext{
38 Dicho de otro modo, la reducción trascendental no es la "eliminación" del mundo natural para quedarse con las meras vivencias de conciencia, así se trate de una conciencia pura. No cabe en fenomenología semejante abstracción. El análisis de las vivencias intencionales supone el contenido específico al que están dirigidas, pero este contenido esasumido en suspensión de su carácter de existencia, es decir, como puro fenómeno de sentido en la experiencia. En sus lecciones de Filosofía primera Husserl repara en el eventual malentendido en la reducción trascendental que podría considerarse como una suerte de autohipnosis donde quedaría "ciego para los objetos". Parecería como si la epojé significara apagar el interruptor del mundo que nos rodea. Pero justo lo que significa la reducción trascendental es todo lo contrario, la reducción nos acerca a la mayor concreción de nuestra propia experiencia en la que
} 
Por otro lado hemos analizado la posibilidad de una ontología trascendental universal que tomaría como hilo conductor los sistemas constitutivos derivados del análisis intencional de las ontologías regionales y formales, así como la explicitación genética de las operaciones implicadas en tales estructuras intencionales. El procedimiento de la reducción trascendental permite tomar las ontologías como lineamientos de orientación que no comprometen el estatuto ontológico de su validez, pues justo ese paso ha sido suspendido por el golpe de la epojé y por otro lado, si la explicitación genética se realiza desde la esfera trascendental evadimos el problema de entenderla como génesis puramente psicológica. Con ello hemos destacado la importancia de la reducción trascendental en la exposición sistemática de la fenomenología como programa y una forma de entender tal reducción, al menos en su acceso a la esfera trascendental a partir del análisis de horizonte.

Con todo, hay problemas de fondo relativos a la propia ejecución de la reducción trascendental y sus alcances efectivos parte del debate actual. Éstos constituyen el principal reto programático de la fenomenología trascendental en nuestros días ${ }^{39}$.

permanecemos, como afirma el propio Husserl, "con los ojos abiertos para todo". "Ich werde also nicht wie durch eine Selbsthypnose objektblind (etwa gar, wenn ich zur Ausschaltung der ganzen Welt übergehe, für diese ganze blind) sorndern für alles bleibe ich sehende". Hua VIII, p. 111.

39 Sobre la crítica a la reducción fenomenológica, véase Hans Rainer Sepp, "Apropiación por desapropiación. Paradojas de la reducción fenomenológica", Escritos de Filosofía 35/36 (1999). En este trabajo Sepp explora la crítica temprana del joven Heidegger y sus alcances en la interpretación de Michel Henry de la epojé fenomenológica. La interpretación que sugiere Sepp en este ensayo supone una renovación de la radicalidad de la reducción fenomenológica a la manera en que la entiende Husserl como un desprendimiento de la subjetividad respecto de su relación empírica con el mundo. Dicho desprendimiento es un ejercicio de reapropiación de sí mismo que surge en posiciones extremas y esta situación representaría una ruptura que emerge de la confrontación con la "facticidad de lo real". Sepp interpreta el acontecimiento de la reducción a la manera de una ruptura. "El análisis fenomenológico del proceso de la epojé debería captar la efectuación de la epojé como una extrema posibilidad de la relación del sí-mismo con el mundo, de tal manera que este caso el sí mismo, confrontado de repente puramente en el modo de la vivencia, con el mundo en cuanto tal, alcanza su relación con el mundo en una situación extrema y experimenta una particular apropiación de sí mismo" (p. 18). Más adelante agrega: "La propia originaria vivencia de una apropiación del sí-mismo en la "profundidad" de la experiencia fenomenológica sería así el fundamento directo para la aclaración fenomenológica de esta experiencia fenomenológica de la profundidad. A partir de aquí se podría hacer también el intento de determina las tareas y límites de una fenomenología de lo subjetivo y asignar su lugar a orientaciones de investigación, como, por ejemplo, la fenomenología genética de Husserl" (idem). El énfasis que sugerimos en el análisis de la implicación intencional de horizonte como hilo conductor hacia el descubrimiento de la esfera trascendental encuentra eco en la propuesta de Sepp de entender la reducción como análisis retrospectivo y su motivo en la ruptura con el mundo fáctico. 